\title{
Cenários de mudanças climáticas e seus impactos na produção leiteira em estados nordestinos
}

\author{
Thieres G. F. da Silva', Magna S. B. de Moura ${ }^{2}$, Ivan I. S. Sá2, Sérgio Zolnier ${ }^{3}$, Sílvia H. N. Turco ${ }^{4}$ \& Luciana S. B. de Souza ${ }^{3}$
}

RESUMO

A pecuária leiteira é uma atividade de grande importância no nordeste brasileiro, podendo ser afetada de forma direta e indireta, caso as atuais previsões climáticas sejam confirmadas até o final do século. 0 objetivo deste trabalho foi estimar os impactos das mudanças climáticas sobre a produção leiteira nos estados de Alagoas, Bahia e Sergipe. Os valores do índice de temperatura e umidade (ITU), do declínio da produção de leite (DPL) e da redução do consumo alimentar (RCA) de vacas leitei ras foram calculados para os cenários climáticos B1 e A1F1 do IPCC. Utilizou-se um modelo multiplicativo para estimar o efeito das alterações de temperatura sobre os valores normais da umidade relativa do ar. Com base em ambos os cenários do IPCC, as regiões produtoras, situadas no norte e litoral do estado da Bahia e áreas litorâneas dos estados de Alagoas e Sergipe, serão afetadas expressivamente em decorrência do aumento do estresse térmico, com reduções marcantes na produção de leite e no consumo alimentar, especialmente de animais com al to potencial genético de produção. Este cenário pode afetar as atuais regiões produtoras dos estados nordestinos, de forma que a exploração leitei ra seja limitada aos animais com baixo desempenho produtivo.

Palavras-chave: conforto térmico, gado leiteiro, ITU, risco climático, SIG

\section{Climate change scenarios and their impacts on milk production in northeastern states of Brazil}

\begin{abstract}
Milk production is an activity of great importance in the Brazilian context, and it may be directly and indirectly affected if current climate predictions are confirmed until the end of this century. The objective of this work was to estimate climate change impacts on milk production in the states of Alagoas, Bahia and Sergipe. Values of the temperature humidity index (THI), decline in milk production (DMP) and decline in feed intake (DFI) of dairy cows were calculated for the B1 and A1F1 climate scenarios, described by the IPCC. A multiplicative model was used to estimate the effect of temperature change on the normal values of relative humidity. Based on both IPCC scenarios, production regions, located in the north and coast of the Bahia state and coastal areas of the states of Alagoas and Sergipe, will be adversely affected due to thermal stress, with profound reduction in milk production and feed intake, especially in case of animals with high genetic production potential. This scenario may affect the current producing in the northeastern states of Brazil, in such a way that milk production be limited only to animals with low productive performance.
\end{abstract}

Key words: thermal comfort, dairy cattle, THI, climatic risk, GIS

\footnotetext{
1 UFRPE/U AST, Fazenda Saco, s/n, Caixa Postal 063, Zona Rural, 56900-000, Serra Talhada, PE. Fone: (87) 3831-1927. E-mail: thieres freire@yahoo.com.br 2 Embrapa Semi-Árido, BR 428, Km 152, CP 23, Zona Rural, CEP 56302-970, Petrolina, PE. Fone: (87) 3862-1711. E-mail: magna@cpatsa.embrapa.br; ighour@cpatsa.embrapa.br

3 DEA/UFV, Av. P. H. Holfs, s/n, Centro, 36570-000, Viçosa - MG.Fone: (31) 3899-1880. E-mail: zolnier@ufv.br, sanddrabastos@hotmail.com

4 UNIVASF, Av. Santo Antonio Carlos M agalhães 510, Bairro Santo Antonio, CEP 48902-300, Juazeiro, BA. Fone: (74) 3612-1135. E-mail: silvia.turco@univasf.edu.br
} 


\section{INTRODUÇÃO}

Acredita-se que a expansão das atividades industriais nas últimas décadas tenha contribuído significativamente para a alteração do clima da Terra. Segundo informações do IPCC (2007), o aumento das concentrações dos gases carbônico, metano e óxido nitroso na atmosfera, é um dos fatores responsáveis pela ocorrência de eventos climáticos extremos, aumento da temperatura global, elevação do nível dos oceanos e intensificação da variabilidade espacial e da magnitude dos valores de precipitação. Com base no relatório do IPCC, alterações da concentração de gás carbônico devem ser atribuídas ao uso de combustíveis fósseis, enquanto as concentrações de metano e de óxido nitroso estão relacionadas à expansão das atividades agropecuárias (IPCC, 2007).

O Brasil, por apresentar a maior parte do seu território situada nas regiões tropicais, possui grande vulnerabilidade às mudanças climáticas, podendo afetar significativamente o agronegócio. A pecuária leiteira é um dos setores mais sensíveis às variações do clima, desempenhando grande importância no contexto nacional. Esta atividade é responsável pela movimentação de 10 bilhões de dólares por ano e emprega cerca de 3 milhões de pessoas (EMBRAPA, 2008).

A pecuária leiteira pode ser afetada, direta e indiretamente pelas mudanças climáticas. Os fatores indiretos estão associados à redução da disponibilidade e ao aumento dos preços de grãos; às alterações na distribuição de pragas e doenças e à diminuição da produção e qualidade de plantas forrageiras (Sirohi \& Michaelowa, 2007). De maneira mais direta, as mudanças no clima podem intensificar o estresse térmico em decorrência de alterações no balanço de energia térmica entre o animal e o ambiente, o qual é influenciado pelos fatores ambientais (radiação, temperatura, umidade relativa e velocidade do vento) e mecanismos de termorregulação (condução, radiação, convecção e evaporação) (Sirohi \& Michaelowa, 2007). Assim, quaisquer alterações nesses fatores podem modificar a zona de termoneutralidade e provocar desconforto para o animal.

Sob condições de estresse térmico, várias alterações metabólicas e comportamentais podem ser induzidas em vacas leiteiras, com destaque para o aumento da temperatura corporal e da frequência respiratória, tal como mudanças no balanço químico corporal dos animais (Srikandakumar \& Johnson, 2004). Adicionalmente, podem ser observadas reduções no consumo alimentar, na reprodução, na gestação, na lactação e, consequentemente, na eficiência produtiva (García-Ispierto et al., 2006; Hahn, 1999; West et al., 2003).

A avaliação do bem-estar animal pode ser realizada por meio de vários índices de conforto ou desconforto térmico, destacando-se o índice de temperatura e umidade do ar (ITU) (Roseler et al., 1997), proposto por Thom (1959). Em avaliações realizadas no interior de instalações agrícolas, este é o índice mais utilizado, haja vista que os efeitos simultâneos da temperatura e umidade relativa do ar no desempenho dos animais são incorporados em um único valor.

O ITU apresenta grande potencial para ser utilizado em trabalhos de avaliação do risco climático (Brown-Brandl et al., 2005; Klosowski et al., 2002) e realização de estudos em esca- la macroclimática (Casa \& Ravelo, 2003; Pires et al., 2006; Somparn et al., 2004; Turco et al., 2006). Outro aspecto favorável à utilização do ITU se deve ao fato deste índice não apresentar, em alguns tipos de instalações, diferenças significativas em relação aos valores do ITGU (Índice de temperatura do globo negro e umidade) (Buffington et al., 1981).

Os impactos das mudanças climáticas globais sobre o rendimento e zoneamentos agroclimáticos das culturas, foram avaliados em diversas pesquisas (Mera et al., 2006; Assad et al., 2004; Marin et al., 2007); entretanto, poucos são os estudos destinados à atividade leiteira (Butt et al, 2005; Olesen \& Bindi, 2002), especialmente em escala macroclimática, nas condições do Brasil.

Uma das grandes dificuldades em se avaliar os impactos das mudanças do clima nos índices relacionados ao bem-estar animal são as incertezas sobre as alterações nos valores de umidade relativa do ar; desta forma, para realização de estudos mais detalhados, Silva et al. (2007a) propõem a utilização de um modelo estatístico com ótimo desempenho e consistência física e, daí, possibilitam o conhecimento dos possíveis efeitos das mudanças climáticas sobre os valores normais da umidade relativa do ar.

Objetivou-se, com este trabalho, determinar os impactos dos cenários climáticos $\mathrm{B} 1$ e A1F1, conforme relatado pelo IPCC, sobre a produção leiteira nos estados de Alagoas, Bahia e Sergipe.

\section{MATERIAL E MÉTODOS}

\section{Especificidades climáticas da região avaliada}

Utilizaram-se, para a realização deste trabalho, dados dos estados de Alagoas, Bahia e Sergipe, os quais respondem por $47,06 \%$ da produção leiteira da região Nordeste do Brasil (IBGE, 2008). Do ponto de vista climático, apresentam grandes variações nas condições de temperatura e umidade relativa do ar devido às diferentes áreas de transições entre o litoral, agreste e semiárido nordestino, resultando, com isto, em distintos ambientes de conforto ou desconforto térmico animal.

\section{Elaboração do banco de dados climáticos}

Foram utilizados os dados históricos mensais de precipitação e os valores observados e estimados de temperatura $\left(\mathrm{t}_{\mathrm{m}}\right)$ e umidade relativa média do ar $\left(\mathrm{UR}_{\mathrm{m}}\right)$, referentes ao período de 1961 a 1990, de 542 localidades, situadas nos estados de Alagoas, Bahia e Sergipe, assim como regiões limítrofes; desse total, 36 são estações meteorológicas principais, vinculadas ao Instituto Nacional de Meteorologia (INMET), e as demais (506) são postos pluviométricos, pertencentes à Rede Hidrometeorológica da Superintendência de Desenvolvimento do Nordeste (SUDENE). O banco de dados climáticos para os postos pluviométricos foi implementado com a utilização de equações de estimativa dos valores históricos normais de temperatura e de umidade relativa do ar.

Os valores normais mensais de temperatura média do ar foram estimados com a utilização de dados geográficos (la- 
titude, $\varphi$; longitude, $\lambda$; altitude, h) dos 506 postos pluviométricos e equações propostas por Cavalcanti \& Silva (1994). Realizou-se a estimativa dos valores normais mensais de umidade relativa do ar com a utilização do modelo multiplicativo proposto por Silva et al. (2007a) para os estados de Alagoas, Bahia e Sergipe.

\section{Estimativa das variações dos valores normais da umidade relativa do ar $(\Delta \mathrm{UR})$}

Em decorrência dos cenários de mudanças climáticas, as variações da umidade relativa do ar ( $\Delta \mathrm{UR})$, foram estimadas por meio do modelo multiplicativo, proposto por Silva et al. (2007a). Esses autores propõem um modelo genérico para estimativa dos valores normais de umidade relativa do ar $\left(\mathrm{UR}_{\mathrm{m}}\right)$ a partir do índice efetivo de umidade $\left(\mathrm{I}_{\mathrm{m}}\right)$, temperatura média do ar $\left(\mathrm{t}_{\mathrm{m}}\right)$ e longitude local $(\lambda)$. $\mathrm{O}$ modelo foi calibrado para os estados de Alagoas, Bahia e Sergipe, para os quais apresentou excelente desempenho estatístico.

$\mathrm{O}$ valor de $\mathrm{I}_{\mathrm{m}}$ representa a disponibilidade hídrica mensal de determinado local, o qual é afetado pelos valores médios mensais de precipitação $\left(\mathrm{P}_{\mathrm{m}}\right)$ e de evapotranspiração $\left(\mathrm{ETP}_{\mathrm{m}}\right)$, obtido a partir da relação $\left[\left(\mathrm{P}_{\mathrm{m}} / \mathrm{ETP}_{\mathrm{m}}\right)^{-1}\right] \times 100$. Com isso, nos períodos de maior e menor disponibilidade hídrica, os valores de umidade relativa do ar tendem a ser maiores e menores, respectivamente (Silva et al., 2007a). Por outro lado, tem-se que a variação da temperatura é inversa à da umidade relativa do ar e que as regiões litorâneas são mais úmidas que as áreas continentais, ou seja, para alguns estados do Nordeste, os dados de umidade relativa do ar diminuem com a longitude (Silva et al., 2007a,b). Assim, por meio do modelo multiplicativo, é possível estimar os valores normais mensais de umidade relativa do ar para qualquer localidade. O modelo é constituído de duas funções, em que a primeira se refere ao efeito do índice $\mathrm{I}_{\mathrm{m}} \mathrm{e}$ a segunda ao efeito da temperatura do ar e da longitude na umidade relativa do ar (UR). Este modelo pode ser escrito como:

Os parâmetros a, b, c, d e e são ajustados regionalmente

$$
\mathrm{UR}=\mathrm{a} \exp \left\{-\exp \left[\frac{-\left(\mathrm{I}_{\mathrm{m}}-\mathrm{b}\right)}{\mathrm{c}}\right]\right\}\left[\mathrm{d}+\mathrm{e}\left(\mathrm{t}_{\mathrm{m}} \lambda\right)\right]
$$

(Silva et al., 2007a) e o símbolo $\lambda$ se refere a longitude que, neste caso, corresponde aos postos de observação pluviométrica.

Os cenários do IPCC (2007) relatam os valores médios das variações de temperatura e precipitação (por exemplo, $1,8^{\circ} \mathrm{C}$ ); com isto, optou-se por estimar os valores médios da $\Delta \mathrm{UR}$ para qualquer localidade e mês do ano de cada um dos três estados avaliados. Para se calcular os valores da $\Delta U R$, primeiro se estimaram os valores futuros de umidade relativa do ar inserindo-se os valores de mudanças climáticas de $\mathrm{t}_{\mathrm{m}}$ no cálculo de $I_{m}$ da primeira função e nos valores de $t_{m}$ na segunda equação do modelo multiplicativo (Eq. 1); em seguida, os resultados foram diminuídos dos valores da umidade relativa do cenário atual para cada mês e localidade, ou seja, $\Delta \mathrm{UR}=\mathrm{UR}_{\text {cenário futuro }}-\mathrm{UR}_{\text {cenário atual }}$.

\section{Cenários de mudanças climáticas}

Neste trabalho foram contemplados dois cenários de mudanças climáticas propostos pelo IPCC (2007) para o ano de 2099, sendo o primeiro considerado otimista (B1) e o segundo pessimista (A1F1). Esses cenários estimam incrementos de 1,8 e $4,0{ }^{\circ} \mathrm{C}$, respectivamente, na temperatura do ar em escala global. Dependendo do cenário adotado, esses incrementos foram adicionados aos valores médios atuais de temperatura mensal $\left(\mathrm{t}_{\mathrm{m}}\right)$ observados ou estimados para cada um dos três meses mais quentes e frios das 542 localidades avaliadas.

Em decorrência de incertezas quanto às variações de precipitação referentes aos cenários B1 e A1F1 para o Nordeste do Brasil, assumiu-se que os valores normais atuais permanecerão inalterados até o final deste século. A partir das projeções futuras de $t_{m}$, foram realizadas as estimativas dos valores normais mensais de umidade relativa para o final deste século, adicionando-se as variações dos valores de umidade relativa do ar ( $\triangle \mathrm{UR})$, obtidos como mencionados anteriormente, aos valores atuais de $\mathrm{UR}_{\mathrm{m}}$ observados ou estimados por meio da Eq. 1. Finalmente, as projeções de $t_{m}$ e $U R_{m}$ foram utilizadas para estimativa dos valores de ITU, DPL e RCA para os cenários $\mathrm{B} 1$ e A1F1 e, posteriormente, comparados com o cenário atual.

\section{Estimativa dos valores de ITU, DPL e RCA}

Os valores de ITU foram obtidos para os três meses considerados mais quentes (dezembro a fevereiro) e mais frios do ano (junho a agosto), que correspondem ao período com maiores e menores possibilidades de declínio da produção de leite, respectivamente.

Observaram-se os valores de temperatura e umidade relativa do ar para esses períodos através da média dos dados dos três meses. Os dados do índice de temperatura e umidade do ar (ITU, adimensional) foram calculados a partir desses valores, utilizando-se a equação proposta por Thom (1959):

$$
\mathrm{ITU}=\mathrm{t}_{\mathrm{m}}+0,36 \mathrm{t}_{\mathrm{po}}+41,5
$$

em que:

$\mathrm{t}_{\mathrm{m}}$ - temperatura média do ar, ${ }^{\circ} \mathrm{C}$

$t_{\mathrm{po}}$ - temperatura do ponto de orvalho, expressa em ${ }^{\circ} \mathrm{C}$, calculada a partir da combinação das equações psicrométricas citadas por Vianello \& Alves (2000):

$$
\mathrm{t}_{\mathrm{po}}=\frac{186,4905-237,3 \log _{10}(\mathrm{UR} \text { es/100) }}{\log _{10}(\mathrm{UR} \text { es } / 100)-8,2859}
$$

em que:

$$
\begin{aligned}
& \text { UR - umidade relativa do ar, \%, obtida pela Eq. } 1 \\
& \text { es - pressão de saturação de vapor d'água no ar, hPa, } \\
& \text { estimada pela expressão: }
\end{aligned}
$$

$$
\text { es }=6,1078 * 10^{\left(\frac{7,5 t}{237,3+t}\right)}
$$

Obteve-se o declínio da produção de leite (DPL) por meio 
da equação proposta por Berry et al. (1964), que fornece os valores de DPL para vacas da raça holandesa como função do ITU e do nível de produção de leite (NP) dos animais:

$$
\mathrm{DPL}=-1,075-1,736 \mathrm{NP}+0,02474 \mathrm{NP} \text { ITU }
$$

em que:

DPL - expresso em kg animal- $\mathrm{d}^{-1}$

$\mathrm{NP}$ - dado em kg animal ${ }^{-1} \mathrm{~d}^{-1}$. O NP é utilizado como referência para representar uma situação em que o animal apresenta produtividade normal sob condições de termoneutralidade. Para este trabalho se consideraram os valores de NP de 10 , 20 e $30 \mathrm{~kg}_{\text {animal }}{ }^{-1} \mathrm{~d}^{-1}$.

Com os valores de ITU também foi quantificada a redução do consumo alimentar (RCA) dos animais leiteiros da raça holandesa, expressa em kg animal ${ }^{-1} \mathrm{~d}^{-1}$, a partir da equação citada por Hahn \& Osburn (1969):

$$
\mathrm{RCA}=-28,23+0,391 \text { ITU }
$$

\section{Espacialização}

Os valores médios de ITU, DPL e RCA para os três meses mais frios e quentes do ano e os dados geográficos das 542 localidades distribuídas por toda área de estudo, foram utilizados na elaboração de mapas que ilustram os efeitos das projeções de mudanças climáticas no desempenho produtivo da atividade leiteira, para os estados de Alagoas, Bahia e Sergipe.

Realizou-se a identificação das áreas adequadas para a produção leiteira de acordo com os valores críticos de ITU, por meio da inversão da Eq. 5, de tal modo que valores nulos de DPL são obtidos quando os valores de ITU são iguais ou inferiores a 74,3, 72,3 e 71,6 para os níveis de produção de 10,20 e $30 \mathrm{~kg}_{\text {animal }}{ }^{-1} \mathrm{~d}^{-1}$, respectivamente; esses valores críticos indicam o início da redução da produção de leite, de acordo com o nível de produção, sendo que os animais mais produtivos são mais sensíveis ao estresse térmico.

Os efeitos das projeções de alterações climáticas na produção de leite foram divididos em classes de ITU, conforme sugerido por Brown-Brandl et al. (2005), sendo que valores de ITU inferiores a 74 caracterizam aproximadamente condições ambientais adequadas para os três níveis de produção $\left(10,20\right.$ e $\left.30 \mathrm{~kg}^{\text {animal }}{ }^{-1} \mathrm{~d}^{-1}\right)$; entre 74 e 78 são observados em ambientes de estresse térmico aceitável e, entre 78 e 84, estabelecem situações de estresse moderado; acima de 84 resultam em estresse térmico severo.

Com os valores críticos de ITU para as vacas leiteiras com os níveis de produção 10,20 e $30 \mathrm{~kg}$ animal $^{-1} \mathrm{~d}^{-1}$ e as classes de estresse térmico citadas por Brown-Brandl et al. (2005), considerou-se o valor 74 como limite para definir as áreas de risco climático para a produção leiteira. Utilizou-se o software ArcGis 9.0, o modelo esférico e o método de interpolação "krigagem ordinária" para obtenção dos mapas de ITU, DPL e RCA dos meses mais quentes e frios do ano no cenário atual e de mudanças climáticas dos Estados de Alagoas, Bahia e Sergipe.

\section{RESULTADOS E DISCUSSÃO}

\section{Impactos das mudanças climáticas sobre os valores de $\triangle \mathrm{UR}$, ITU, DPL e RCA}

Com base no aumento da temperatura média do ar em $1,8{ }^{\circ} \mathrm{C}$ até o final deste século, conforme projetado pelo cenário climático $\mathrm{B} 1$, os valores normais de umidade relativa do ar, estimados de acordo com equação proposta por Silva et al. (2007a), sofrerão reduções de 4,6 $\pm 0,59 \%$, $4,5 \pm 0,49 \%$ e $4,4 \pm 0,52 \%$ para os estados de Alagoas, Bahia e Sergipe, respectivamente; entretanto, a partir de projeções mais pessimistas (A1F1), estima-se que essas reduções serão ainda maiores, atingindo $10,2 \pm 1,52 \%$, $10,1 \pm 1,26 \%$ e $10,2 \pm 1,07 \%$, respectivamente. Observouse que o modelo proposto por Silva et al. (2007a) apresentou consistência quanto aos resultados obtidos, pois o aumento da temperatura do ar sem variações nos valores de precipitação, resultou em reduções equivalentes nos valores de umidade relativa do ar.

Combinando-se o aumento dos valores de $t_{\mathrm{m}}$ com as reduções de $\mathrm{UR}_{\mathrm{m}}$, observaram-se alterações expressivas nas áreas, com possibilidades de criação de vacas leiteiras, sobretudo para os meses mais quentes do ano (dezembro a fevereiro).

Apresentam-se, na Figura 1, as variações regionais dos valores de ITU para o cenário atual e para as duas projeções de mudanças climáticas. Observou-se, no cenário atual (Figura $1 \mathrm{~A}$ e B), que na maior parte da área estudada $(53,95 \%)$ os valores de ITU, tanto para os meses mais quentes como para os mais frios, foram inferiores a 74; este valor estabelece o limite crítico acima do qual as condições climáticas podem reduzir o desempenho produtivo dos animais, caso técnicas de manejo não sejam adotadas.

Áreas com ITU < 74, que corresponderam à maior parte do Estado da Bahia, são caracterizadas como satisfatórias para criação de vacas leiteiras, segundo a classificação de Brown-Brandl et al. (2005). As áreas com as melhores condições para criação de animais especializados para o setor leiteiro, com produção de $30 \mathrm{~kg}_{\text {animal }}{ }^{-1} \mathrm{~d}^{-1}$, tanto para os meses frios quanto para os mais quentes, estão situadas nas regiões central e centro sul do Estado (Figura 1A, B), e respondem por $8,60 \%$ do território em estudo.

Condições de estresse térmico ameno, com valores de ITU variando entre 74 e 75 , podem ser constatadas nas principais mesorregiões dos Estados de Alagoas (Santana do Ipanema, Batalha, Palmeira dos Índios e Arapiraca), Bahia (Porto Seguro) e Sergipe (Sergipana do Sertão do São Francisco) (IBGE, 2008).

Com as mudanças climáticas, o cenário mais otimista (B1) reduziu as áreas adequadas (ITU < 74) para 8,04\% nos meses mais quentes (Figura 1C); nos meses mais frios (Figura 1D), apesar das mudanças climáticas, constatou-se que grandes extensões dos estados $(84,94 \%)$ apresentaram condições favoráveis, contudo verifica-se um aumento dos valores de ITU, que ficaram entre 72 e 74 em várias áreas, o que pode afetar o desempenho de animais mais especializados na produção de leite $\left(30 \mathrm{~kg}_{\text {animal }}{ }^{-1} \mathrm{~d}^{-1}\right)$.

Durante os meses mais quentes, a maior parte da região 

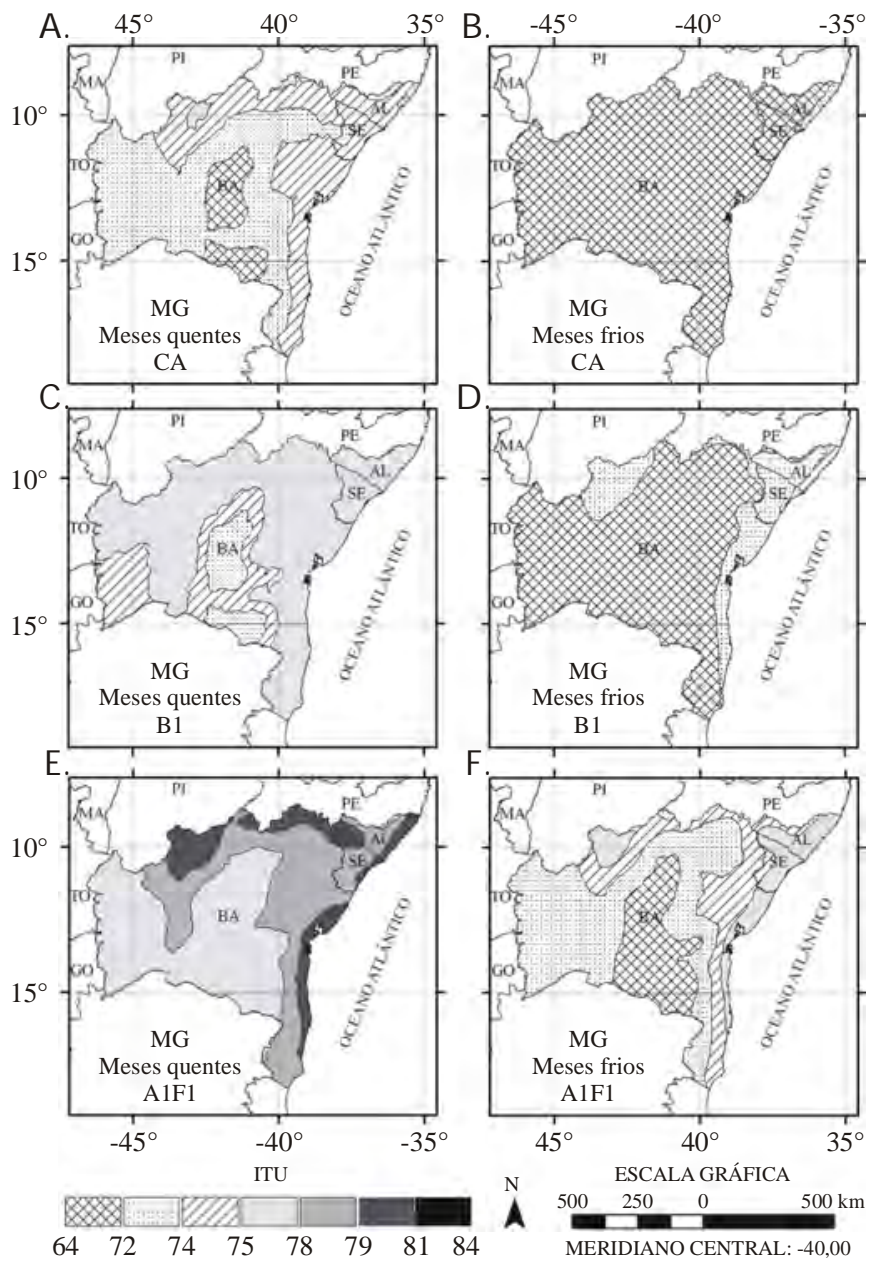

DATUM: WGS_84

Figura 1. Variação regional dos valores médios do índice de temperatura e umidade do ar (ITU), para os três meses mais quentes ( $A, C$ e $E$ ) e mais frios do ano $(B, D$ e $F)$ da área em estudo, considerando-se o cenário atual - CA (A e B) e cenários futuros B1 (C e D) e A1F1 (E e F)

de estudo $(78,11 \%)$ foi caracterizada com estresse térmico ameno, inclusive para as atuais mesorregiões leiteiras situadas nos estados de Alagoas, Bahia e Sergipe, nas quais o estresse térmico foi intensificado (ITU entre 75 e 78), quando comparado com o cenário atual (ITU entre 74 e 75); assim, técnicas de manejo mais sofisticadas deverão ser adotadas visando reduzir a perda de rendimento dos animais.

As maiores reduções ocorreram no cenário mais pessimista (A1F1) (Figuras 1E e F). Nos meses mais quentes, as regiões mais afetadas (ITU entre 79 e 81) se situaram ao norte e litoral do estado da Bahia e nas regiões litorâneas dos estados de Alagoas e Sergipe. As áreas caracterizadas por estresse moderado para produção leiteira (ITU variando entre 78 e 84 ) respondem por $45,44 \%$ do território estudado.

Nas Figuras 2 e 3 se encontram as variações regionais dos valores DPL para vacas leiteiras com níveis de produção de 10,20 e $30 \mathrm{~kg}$ de leite animal ${ }^{-1} \mathrm{~d}^{-1}$ durante os meses mais quentes e frios dos cenários atual (CA) e de mudanças climáticas (B1 e A1F1). Os valores nulos de DPL durante o meses mais quentes do cenário atual foram observados na maior parte dos Estados avaliados para animais menos especializados (10 kg de leite animal ${ }^{-1} \mathrm{~d}^{-1}$ ) (Figura 2A, 2D e 2G).
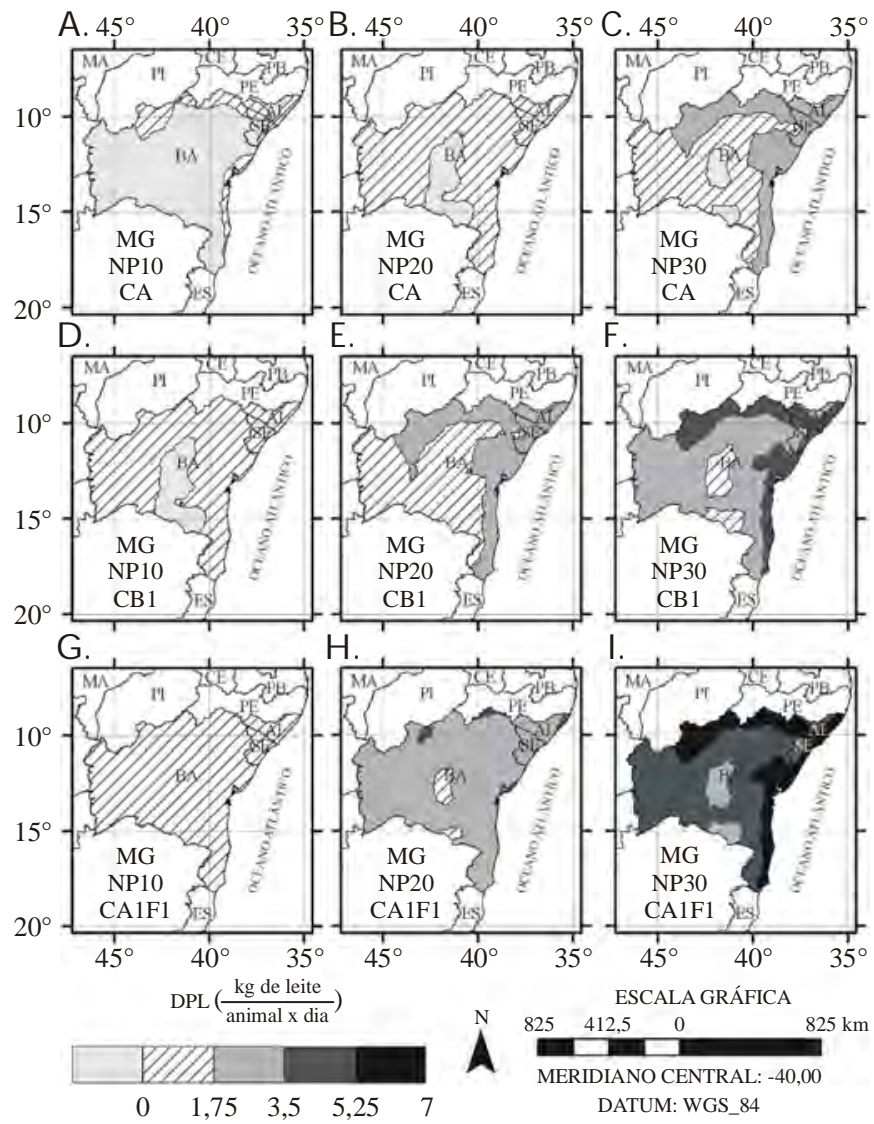

Figura 2. Variação regional dos valores médios do declínio da produção de leite (DPL), para animais com níveis de produção (NP) de 10 (A, D e $\mathrm{G}), 20\left(\mathrm{~B}, \mathrm{E}\right.$ e H) e $30 \mathrm{~kg}^{2}$ animal ${ }^{-1} \mathrm{dia}^{-1}(\mathrm{C}, \mathrm{Fe} \mathrm{I})$, nos meses mais quentes da área em estudo, considerando-se o cenário atual $(A, B$ e $C)$ e cenários futuros $B 1$ (D, E e F) e AlF1 (G, H e I)

As maiores perdas de produção de leite foram observadas para animais com níveis de 20 e $30 \mathrm{~kg}$ de leite animal ${ }^{-1} \mathrm{~d}^{-1}$; por outro lado se constataram, para os meses mais frios (Figura 3A, D e G), apenas reduções na produção em áreas litorâneas dos estados de Alagoas e Sergipe para animais mais especializados (30 kg de leite animal-1 $\left.\mathrm{d}^{-1}\right)$; nos meses mais quentes do cenário B1 (Figura 2B, E e H), as áreas com valores de DPL de até 1,75 e $3,5 \mathrm{~kg}_{\text {animal }}{ }^{-1} \mathrm{~d}^{-1}$ aumentaram significativamente para animais de 10 e $20 \mathrm{~kg}$ de leite animal ${ }^{-1} \mathrm{~d}^{-1}$, respectivamente.

Áreas situadas ao norte e litoral do estado da Bahia e na região litorânea dos demais Estados apresentaram reduções de até $3,5 \mathrm{~kg}$ de leite animal ${ }^{-1} \mathrm{~d}^{-1}$ para os animais com NP de $30 \mathrm{~kg}_{\text {animal }}{ }^{-1} \mathrm{~d}^{-1}$ durante os meses mais frios (Figura $3 \mathrm{H}$ ); nos meses mais quentes, essas perdas de produção atingiram valores de até $5,25 \mathrm{~kg}_{\text {animal }}{ }^{-1} \mathrm{~d}^{-1}$.

No cenário mais pessimista (A1F1), o DPL atingiu $7,0 \mathrm{~kg}$ animal ${ }^{-1} \mathrm{~d}^{-1}$ para vacas com NP de $30 \mathrm{~kg}^{\text {animal }}{ }^{-1} \mathrm{~d}^{-1}$, durante o período dos meses mais quentes (Figura 2I); nos meses mais frios se constataram valores de DPL de até $5,25 \mathrm{~kg}_{\text {animal }}{ }^{-1} \mathrm{~d}^{-1}$; referidos declínios de produção foram observados em áreas situadas ao norte e litoral da Bahia e regiões do litoral dos estados de Alagoas e Sergipe (Figura 3I).

Para as vacas leiteiras com NP igual a $10 \mathrm{~kg}_{\text {animal }}{ }^{-1} \mathrm{~d}^{-1}$, os valores de DPL durante os meses mais quentes atingiram 


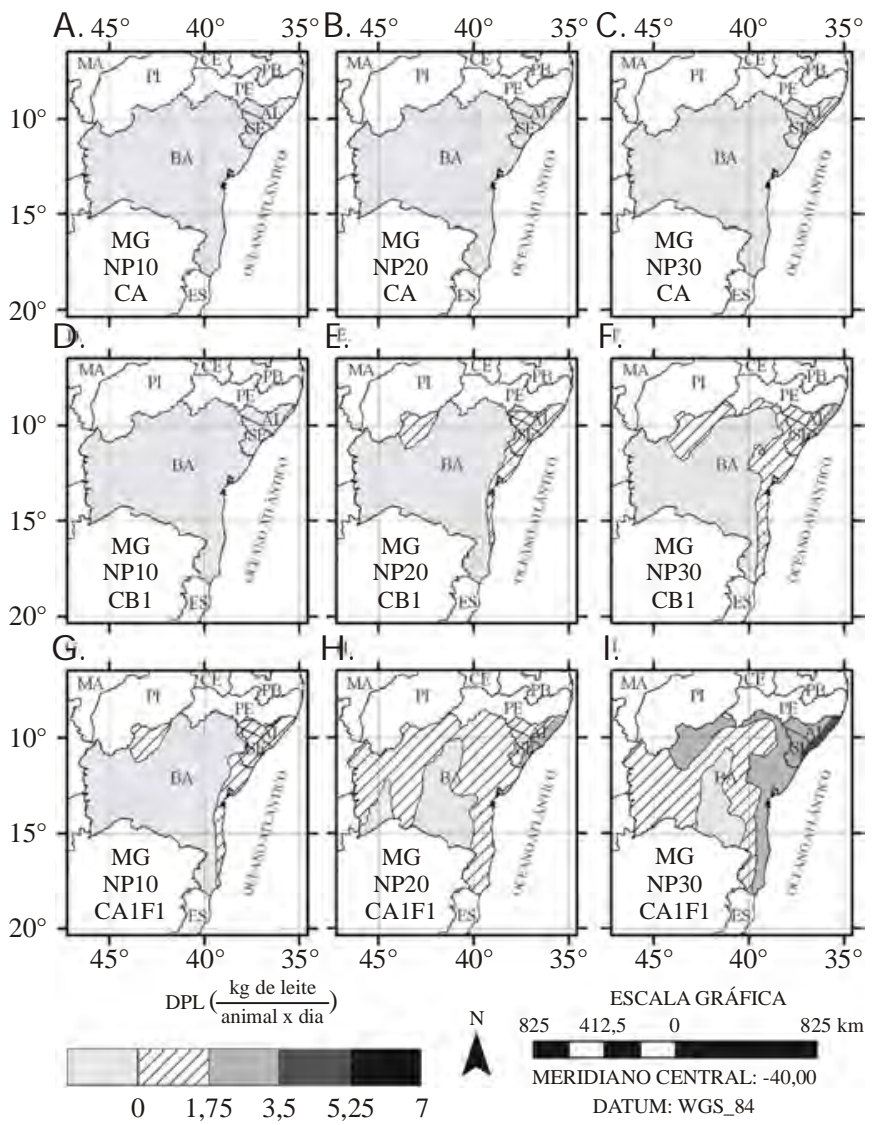

Figura 3. Variação regional dos valores médios do declínio da produção de leite (DPL), para animais com níveis de produção (NP) de 10 ( $A, D$ e $\mathrm{G}), 20$ ( $\mathrm{B}, \mathrm{E}$ e H) e $30 \mathrm{~kg}^{2}$ animal ${ }^{-1} \mathrm{dia}^{-1}(\mathrm{C}, \mathrm{F}$ e I), nos meses mais frios da área em estudo, considerando-se 0 cenário atual $(A, B$ e $C)$ e cenários futuros $B 1$ (D, E e F) e AlF1 (G, H e I)

$1,75 \mathrm{~kg}$ animal ${ }^{-1} \mathrm{~d}^{-1}$ (Figura 2A). Os resultados mostraram que as melhores épocas para a exploração leiteira foram os meses mais frios, logo que a maior parte do terrritório dos Estados apresentou DPL nulo (Figura 3A). Para as vacas com NP de $20 \mathrm{~kg}$ animal ${ }^{-1} \mathrm{~d}^{-1}$ durante os meses mais frios, as maiores reduções de produção ocorreram em áreas situadas no litoral dos Estados (Figura 3B), atingindo valores de até $3,5 \mathrm{~kg}$ animal ${ }^{-1} \mathrm{~d}^{-1}$; essas reduções foram constatadas em maior parte do território, nos meses mais quentes (Figura 2B).

A variação regional dos valores de RCA dos animais nos cenários atual e de mudanças climáticas, é observada na Figura 4; percebe-se que, nos meses quentes do cenário atual (CA), os animais apresentaram valores de RCA de até $1,40 \mathrm{~kg}^{2}$ animal ${ }^{-1} \mathrm{~d}^{-1}$ (Figura 4A), enquanto nos meses mais frios essas reduções atingiram até $0,70 \mathrm{~kg}$ animal ${ }^{-1} \mathrm{~d}^{-1} \mathrm{em}$ áreas situadas na região litorânea (Figura 4B), porém no cenário B1 os valores de RCA atingiram até 0,7 e $2,1 \mathrm{~kg}^{\text {animal }}{ }^{-1} \mathrm{~d}^{-1}$ nos meses mais frios e quentes, respectivamente (Figura 4C e D).

No cenário A1F1 os valores de RCA atingiram até $3,5 \mathrm{~kg}_{\text {animal }}{ }^{-1} \mathrm{~d}^{-1}$ durante os meses mais quentes; essas áreas compreendem o norte do estado da Bahia e regiões litorâneas dos estados de Alagoas e Sergipe (Figura 4E); já nos meses mais frios, tais reduções foram de ordem de $2,1 \mathrm{~kg}^{\text {animal }}{ }^{-1} \mathrm{~d}^{-1}$ (Figura 4F); entretanto, com as mudanças climáticas os efeitos sobre desempenho produtivo dos animais leiteiros podem ser ainda maiores, especialmente para aqueles com melhores rendimentos, uma vez que os mesmos poderão ser submetidos, por períodos prolongados, a condições de estresse térmico, devido à ocorrência de eventos extremos de ondas de calor. West et al. (2003) verificaram que durante dois dias consecutivos de valores de ITU acima de 72,1, vacas leiteiras das raças holandesa e Jersey, apresentaram um DPL de 0,88 e $0,60 \mathrm{~kg}$ por cada unidade de ITU excedida, enquanto a RCA foi de 0,51 e $0,47 \mathrm{~kg}$, respectivamente; tendência similar foi constatada por Ravagnolo et al. (2000), quando submeteram vacas da raça holandesa a valores de ITU superiores a 72, observando DPL de $0,2 \mathrm{~kg}$ por unidade de ITU excedida, e por Campos et al. (2001) que, durante os meses mais quentes do ano, verificaram reduções entre 6,0 e $6,8 \mathrm{~kg}$ de leite por dia.

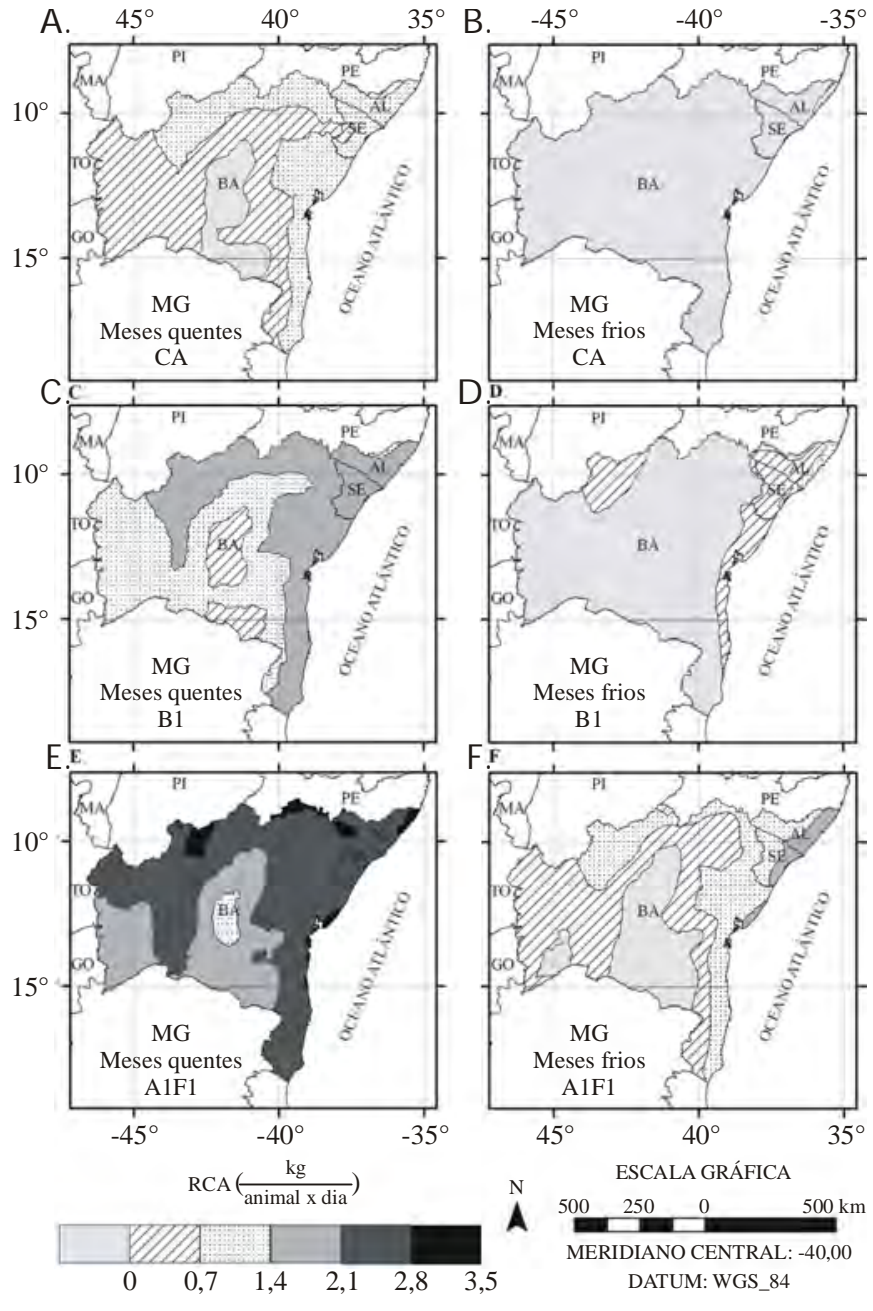

Figura 4. Variação regional dos valores médios da redução do consumo alimentar (RCA), para os meses mais quentes ( $A, C$ e $E$ ) e mais frios do ano $(B, D$ e $F)$ da área em estudo, considerando-se os cenários atual $(A$ e $B)$ e futuros B1 (C e D) e A1F1 (E e F)

\section{Ações mitigadoras dos efeitos das mudanças climáticas sobre a produção leiteira nos Estados analisados}

Diante das possibilidades de mudanças no clima da Terra, técnicas mitigadoras, como modificação física do ambiente, melhorias no sistema de manejo nutricional dos animais e desenvolvimento de raças menos sensíveis ao estresse térmico, deverão 
ser adotadas visando minimizar as projeções de intensificação do estresse térmico animal (Kadzere et al., 2002; West, 2003). Nos ambientes com condições menos estressantes, a utilização de sombra natural ou artificial que, em geral, apresenta custos relativamente baixos, é uma técnica que pode ser adotada para mitigar os efeitos do estresse térmico (Baccari Junior, 1998).

Guiselini et al. (1999) revisaram a influência de vários tipos de sombreamento sobre o conforto térmico de gado leiteiro e constataram que, em alguns casos, a utilização de estruturas mais simples, como sombra de árvores, não apresentaram diferenças significativas em relação a estruturas artificiais e mais sofisticadas como, por exemplo, estábulos com paredes de alvenarias, cobertos com telhas de barro e pé-direito e do cocho coberto com telhas de amianto, aberto e bem ventilado. Em experimentação, esses autores concluíram que o Bambu possui qualidade térmica de sombra superior a outras espécies, como Santa Bárbara, Chapéu de Sol e Leucena, respectivamente.

Dependendo do sistema de produção, a ausência de árvores na propriedade pode ser contornada pelo sombreamento artificial, mediante o uso de sombras portáteis ou de abrigos permanentes, os quais podem ter maior contribuição para animais sob condições mais estressantes (Brown-Brandl et al., 2005).

Nos ambientes com condições muito estressantes para as vacas leiteiras confinadas, o uso de técnicas de climatização como, por exemplo, sistemas de resfriamento adiabático-evaporativo (SRAE), pode constituir-se em alternativa viável para mitigar o estresse animal por calor (Mader et al., 2007), especialmente para animais com elevados índices de produção. O SRAE permite aumentar o conforto térmico e melhorar a produção e qualidade do leite, principalmente quando dietas adequadas são formuladas para os animais durante os períodos mais quentes. Mesmo quando aplicado por períodos curtos, o SRAE reduz os efeitos do estresse sobre as características fisiológicas dos animais (Gallardo et al., 2005).

O uso do SRAE reduz a taxa respiratória e a temperatura retal dos animais e, consequentemente, favorece um aumento de desempenho produtivo (produção leiteira, nível de gordura do leite, eficiência de ganho, entre outros) e reprodutivo (serviços por concepção) após o período de parto (Avendaño-Reyes et al., 2006); entretanto, o uso desse tipo de sistema nos estados estudados deve ser bem planejado visando não elevar muito os gastos de produção.

$\mathrm{O}$ aumento do estresse térmico poderá resultar em perda acentuada de nutrientes do organismo dos animais em decorrência da redução do consumo de matéria seca ou por aumento da taxa de suor; desta forma, a suplementação alimentar torna-se necessária visando, principalmente, beneficiar a produção de leite do animal em áreas com condições climáticas mais quentes (Granzin \& Gaughan, 2002).

A transferência parcial da alimentação para o período noturno de modo que os animais diminuam os gastos de energia durante a alimentação no período diurno, pode reduzir o declínio da produção de leite devido ao estresse e aumentar a eficiência de utilização de energia para a produção leiteira; adicionalmente, o fornecimento abundante de água de boa qualidade durante o período diurno, também pode auxiliar na dissipação de calor pelo animal e redução dos efeitos do estresse térmico (Aharoni et al., 2005).

\section{CONCLUSÕES}

1. Caso as projeções de mudanças climáticas sejam confirmadas até o final deste século, tanto o cenário otimista (B1) como o pessimista (A1F1) demonstrarão que haverá impactos negativos na atividade leiteira nos estados de Alagoas, Bahia e Sergipe.

2. Estimativas do índice de temperatura e umidade sugerem que o estresse térmico será intensificado nos três estados, tanto nos meses mais quentes (dezembro a fevereiro) como nos mais frios (junho a agosto).

3. Se as projeções mais pessimistas forem confirmadas, as mudanças climáticas terão impactos expressivos nos animais mais especializados para o setor leiteiro, com níveis de produção em torno de $30 \mathrm{~kg}$ de leite animal ${ }^{-1} \mathrm{~d}^{-1}$; neste caso a exploração leiteira poderá limitar-se a animais com baixos níveis de produção $\left(10 \mathrm{~kg}\right.$ de leite animal $\left.{ }^{-1} \mathrm{~d}^{-1}\right)$ por serem menos sensíveis ao estresse térmico.

\section{LITERATURA CITADA}

Aharoni, Y.; Brosh, A.; Harare, Y. Night feeding for high-yielding dairy cows in hot weather: effects on intake, milk yield and energy expenditure. Livestock Production Science, v.92, p.207-219, 2005.

Assad, E. D.; Pinto, H. S.; Zullo Junior, J.; Ávila, A. M. H. Impacto das mudanças climáticas no zoneamento agroclimático do café no Brasil. Pesquisa Agropecuária Brasileira, v.39, n.11, p.1057-1064, 2004.

Avendaño-Reyes, L.; Alvarez-Valenzuela, F. D.; Correa-Calderón, A.; Saucedo-Quintero, J.S.; Robinson, P.H.; Fadel, J.G. Effect of cooling Holstein cows during the dry period on postpartum performance under heat stress conditions. Livestock Production Science, v.105, p.198-206, 2006.

Baccari Júnior, F. Manejo ambiental para produção de leite em climas quentes. In: Congresso Brasileiro de Bioclimatologia, 2., 1998, Goiânia. Anais... Goiânia: Sociedade Brasileira de Biometeorologia, 1998. p.136-61.

Berry, I. L.; Shanklin, M. D.; Johnson, H. D. Dairy shelter design based on milk production decline as affected by temperature and humidity. Transactions of the ASAE, v.3, p.329-331, 1964.

Brown-Brandl, T. M.; Eigenberg, R. A.; Nienaber, J. A.; Hahn, G. L. Dynamic response indicators of heat stress in shaded and non-shaded feedlot cattle, Part 1: Analyses of Indicators. Biosystems Engineering, v.90, n.4, p.451-462, 2005.

Buffington, D. E.; Collazo-Arocho, A.; Canton, G. H. Black globehumidity comfort index (BGHI) as comfort equation for dairy cows. Transactions of the ASAE, v.24, n.4, p.711-714, 1981.

Butt, T. A.; McCarl, B. A.; Angerer, J.; Dyke, P. T.; Stuth, J. W. The economic and food security implications of climate change in Mali. Climatic Change, v.68, p.355-378, 2005.

Campos, A. T. de; Pires, M. F. A.; Verneque, R. S.; Campos, A. T.; Campos, D. S. Prognóstico de declínio na produção de leite em função do clima para a região de Goiânia, GO. In: Reunião Anual da Sociedade Brasileira de Zootecnia, 38, 2001, Piracicaba. Anais... Piracicaba: FEALQ, 2001. p.11-13. 
Casa, A. C. de la; Ravelo, A. C. Assessing temperature and humidity conditions for dairy cattle in Córdoba, Argentina. International Journal Biometeorology, v.48, p.6-9, 2003.

Cavalcanti, E. P.; Silva, E. D. V. Estimativa da temperatura do ar em função das coordenadas locais. In: Congresso Brasileiro de Meteorologia, 7, e Congresso Latino-Americano e Ibérico de Meteorologia, 1994, Belo Horizonte. Anais..., Belo Horizonte: Sociedade Brasileira de Meteorologia, 1994. p.154-157.

EMBRAPA - Empresa Brasileira de Pesquisa Agropecuária. Sistema de produção de leite. Embrapa Gado de Leite, Juiz de Fora, MG. http://sistemasdeproducao.cnptia.embrapa.br/FontesHTML/Leite/LeiteCerrado/index.html. 19 Fev. 2008.

Gallardo, M. R.; Valtorta, S. E.; Leva, P. E.; Gaggiotti, M. C.; Conti, G. A.; Gregoret, R. F. Diet and cooling interactions on physiological responses of grazing dairy cows, milk production and composition. International Journal Biometeorology, v.50, p.90-95, 2005.

García-Ispierto, I.; López-Gatius, F.; Santolaria, P.; Yaniz, J. L.; Nogareda, C.; López-Béjar, M.; De Rensis, F. Relationship between heat stress during the peri-implantation period and early fetal loss in dairy cattle. Theriogenology, v.65, p.799-807, 2006.

Granzin, B. C.; Gaughan, J. B. The effect of sodium chloride supplementation on the milk production of grazing Holstein Friesian cows during summer and autumn in a humid sub-tropical environment. Animal Feed Science and Technology, v.96, p.147-160, 2002.

Guiselini, C.; Silva, I. J. O.; Piedade, S. M. Avaliação da qualidade do sombreamento arbóreo no meio rural. Revista Brasileira de Engenharia Agrícola e Ambiental, v.3, n.3, p.380-384, 1999.

Hahn, G. L. Dynamic responses of cattle to thermal heat loads. Dairy Science, v.82 (Suplemento, 2), p.10-20. 1999.

Hahn, G. L.; Osburn, D. D. Feasibility of Summer environmental control for dairy cattle based on expected production losses. Transactions of the ASAE, v.12, n.4, p.448-451, 1969.

IBGE - Instituto Brasileiro de Geografia e Estatística. Produção pecuária 2006. IBGE, Rio de Janeiro. HTTP://www.ibge.gov.br. 19 Fev. 2008.

IPCC - Intergovernmental Painel Climate Change. Climate change 2007: The physical science basis, summary for policymakers. Geneva: IPCC, 2007. 18p.

Kadzere, C. T.; Murphy, M. R.; Silanikove, N.; Maltz, E. Heat stress in lactating dairy cows: A review. Livestock Production Science, v.77, p.59-91, 2002.

Klosowski, E. S; Campos, A. T.; Campos, A. T; Gasparino, E. Estimativa do declínio na produção de leite, em período de verão, para Maringá-PR. Revista Brasileira de Agrometeorologia, v.10, n.2, p.283-288, 2002.

Mader, T. L.; Davis, M. S.; Gaughan, J. B. Effect of sprinkling on feedlot microclimate and cattle behavior. International Journal Biometeorology, v.51, p.541-551, 2007.

Marin, F. R; Assad, E. D.; Barbarisi, B. F.; Pilau, F. G.; Pacheco, L. R. F.; Júnior, J. Z.; Pinto, H. S. Efeito das mudanças climáticas sobre a aptidão climática para cana-de-açúcar no estado de São Paulo. In: Congresso Brasileiro de Agrometeorologia, 15, Aracaju. Anais... Aracaju: Sociedade Brasileira de Agrometeorologia e Embrapa Tabuleiros Costeiros. 2007. CD-Rom.
Mera, R. J; Niyogi, D.; Buol, G. S; Wilkerson, G. G.; Semazzi, F. H. M. Potential individual versus simultaneous climate change effects on soybean $(\mathrm{C} 3)$ and maize $(\mathrm{C} 4)$ crops: An agrotechnology model based study. Global and Planetary Change, v. 54, p.163-182, 2006.

Olesen, J. E.; Bindi, M. Consequences of climate change for European agricultural productivity, land use and policy. European Journal of Agronomy, v.16, p.239-262, 2002.

Pires, M. de F. A.; Silva Junior, J. L. C. da; Campos A. T. de; Costa, L. C.; Novaes, L. P. Zoneamento da região sudeste do Brasil, utilizando o índice de temperatura e umidade. Juiz de Fora: Embrapa Gado de Leite, 2003. 21p. Boletim de Pesquisa, 13

Ravagnolo, O.; Misztal, I.; Hoogenboom, G. Genetic component of heat stress in dairy cattle, development of heat index function. Journal Dairy Science, v.83, p.2120-2125, 2000.

Roseler, D. K.; Fox, D. G. ; Chase, L. E.; Pell, A. N.; Stone, W. C. Development and Evaluation of Equations for Prediction of Feed Intake for Lactating Holstein Dairy Cows. Journal Dairy Science, v.80, p.878-893, 1997.

Silva, T. G. F. da; Zolnier, S.; Moura, M. S. B. de; Sediyama, G. C. Estimativa e espacialização da umidade relativa do ar para os estados de Alagoas, Bahia e Sergipe. Revista Brasileira de Agrometeorologia, v 15, n.1, p.14-28, 2007a.

Silva, T. G. F.; Zonier, S.; Moura M. S. B. de; Sediyama, G. C.; Souza, L. S. B. Umidade relativa do ar: estimativa e espacialização para o estado de Pernambuco. In: Congresso Brasileiro de Agrometeorologia, 15, Aracaju. Anais... Aracaju: Sociedade Brasileira de Agrometeorologia e EMBRAPA/Tabuleiros Costeiros. 2007b. CD-Rom

Sirohi, S.; Michaelowa, A. Sufferer and cause: Indian livestock and climate change. Climatic Change, v.100, p.120-134, 2007.

Somparn, P.; Gibb, M. J.; Markvichitr, K.; Chaiyabutr N.; Thummabood, S.; Vajrabukka, C. Analysis of climatic risk for cattle and buffalo production in northeast Thailand. International Journal Biometeorology, v.49, p.59-64, 2004.

Srikandakumar, A.; Johnson, E. H. Effect of heat stress on milk production, rectal temperature, respiratory rate and blood chemistry in Holstein, Jersey and Australian milking zebu cows. Tropical Animal Health and Production, v.36, p.685-692, 2004.

Thom, E. C. The discomfort index. Weatherwise, v.12, p.57-60, 1959.

Turco, S. H. N.; Silva, T. G. F. da; Santos, L. F. C. dos; Ribeiro, P. H. B.; Araújo, G. G. L.; Júnior, E. V. H.; Aguiar, M. A. Zoneamento bioclimático para vacas leiteiras no estado da Bahia. Revista de Engenharia Agrícola, v.26, n.1, p.20-27, 2006.

Vianello, R. L.; Alves, A. R. Meteorologia básica e aplicações. Viçosa: UFV, 2000. 448p.

West, J. W. Effects of Heat-Stress on Production in Dairy Cattle. Journal Dairy Science, v.86, p. 2131-2144, 2003.

West, J. W.; Mullinix, B. G.; Bernard, J. K. Effects of Hot, Humid Weather on Milk Temperature, Dry Matter Intake, and Milk Yield of Lactating Dairy Cows. Journal Dairy Science, v.86, p.232-242, 2003. 